\title{
Pseudo-dermal sinus tract or spinal dermal-sinus-like stalk?
}

\author{
E. M. J. Cornips • J. W. Weber • J. S. H. Vles • \\ J. van Aalst
}

Received: 17 May 2011 / Accepted: 31 May 2011 /Published online: 8 June 2011

(C) The Author(s) 2011. This article is published with open access at Springerlink.com

\section{Dear Editor,}

We read with great interest the paper "Spinal dermal sinus and pseudo-dermal sinus tracts: two different entities" by Martinez-Lage et al. [1]. In fact, the term "sinus" may be used for (chronically) infected tracts connecting a deepseated infection to the skin, and discharging pus to the surface, except for those tracts connecting two epithelialized surfaces, which are called "fistula".

A spinal dermal sinus (DS) is a developmental anomaly of the dorsal midline axis, in which a hollow, epitheliumlined tract extends inward from the skin surface for a variable distance [1-3]. A key feature of a DS is an open skin defect; however, this may be minute and, as such, easily overlooked during routine neonatal examination [1]. Occasional discharge from this orifice is a common finding, however, even in case of connection with the spinal cord, this never is cerebrospinal fluid [3, 4]. Another key feature of a DS is the accumulation of (epi)dermoid remnants, often presenting as tumors inside the tract or even inside the spinal cord. Such tumors may produce manifestations of spinal cord or cauda equina compression [1]. Moreover,

E. M. J. Cornips $(\bowtie) \cdot$ J. van Aalst

Department of Neurosurgery,

Maastricht University Medical Center (MUMC+),

P. Debyelaan 25, PO Box 5800,

6202 AZ Maastricht, The Netherlands

e-mail: erwincornips@yahoo.com

J. W. Weber $\cdot$ J. S. H. Vles

Department of Child Neurology,

Maastricht University Medical Center (MUMC+),

P. Debyelaan 25, PO Box 5800,

6202 AZ Maastricht, The Netherlands due to an open connection with the skin surface, many cases present with meningitis and intraspinal, intramedullary, or more superficial intra-epidermoid abscesses, a potentially life-threatening condition, rather than with cord tethering (recurrent cases no longer connected to the skin surface) $[1,3]$.

A spinal dermal-sinus-like stalk (DSS) [4] or pseudodermal sinus tract as proposed by Martinez-Lage et al. [1] is a developmental anomaly of the dorsal midline axis, in which a solid tract extends outward from the intradural space to the skin. Key features of a DSS are a closed skin defect (a dimple, cigarette burn, or blister, all without an orifice) usually more obvious than in case of a DS, the absence of a lumen, and the absence of (epi)dermoid remnants inside the tract $[1,4]$. Consequently, a DSS will never present with meningitis, but rather with cord tethering $[1,4]$. To illustrate this point, we have included several photographs highlighting the essential surgical steps in case of a DSS, which, in this particular case, was attached to a low-lying conus medullaris (Fig. 1a-f).

In case of a DS, stratified squamous epithelium inside the lumen, surrounded by dermal tissue, suggests a nondisjunction of cutaneous and neural ectoderm with inward dragging of the epidermis. In case of a DSS, however, mesenchymal and neural elements inside the stalk suggest an embryologic development in the opposite direction (inside-out rather than outside-in). This would explain the absence of a skin orifice, the occasional dorsal tenting of the dura, and the presence of glioneural tissue inside the stalk. Thus, a DSS would represent an atrophic mesodermal-neural stalk [4] and, as suggested by MartinezLage et al., may indeed represent the spinal counterpart of an atretic encephalocele [1]. 

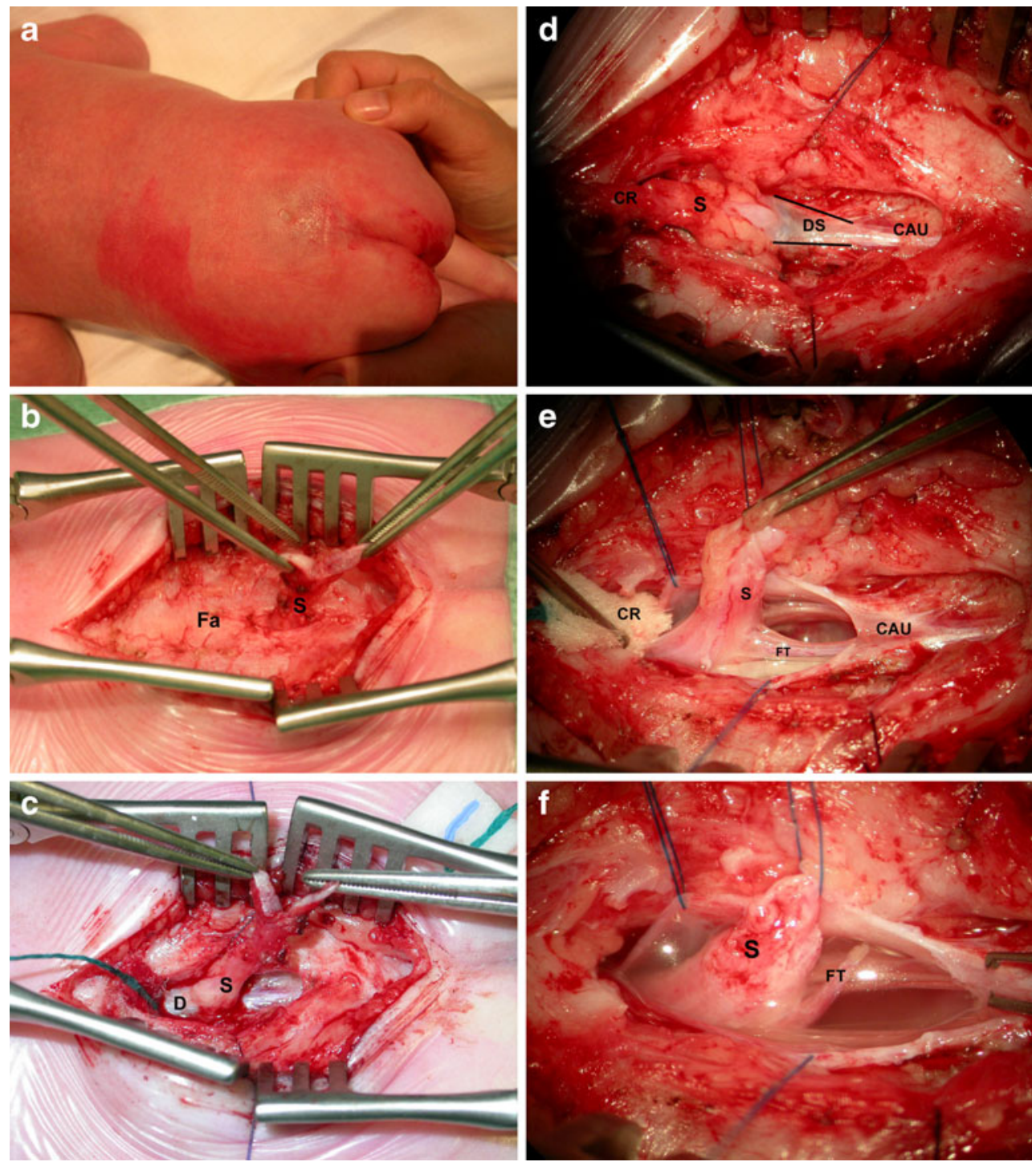

Fig. 1 a-f Illustrates a typical DSS case born at a time DS and DSS were not yet recognized as different entities. The child was scheduled for early operation which, for unrelated causes, was deferred until the age of 3 months. Essential surgical steps are illustrated. CAU caudal, $C R A$ cranial, $D$ dorsal, $D S$ dural sleeve, $F a$ lumbar fascia, $F T$ filum terminale, $S$ stalk. a Skin defect (dimple) typically seen in case of a DSS: above the gluteal fold, in the midline, and without an orifice. $\mathbf{b}$ Dimple and surrounding skin are excised, and the attached stalk is followed through the lumbar fascia to its ending, which in case of a DSS should be intradurally. $\mathbf{c}$ The stalk is followed to the point where

In conclusion, DS and DSS are clearly different clinicopathological entities that share some common cutaneous and neuro-imaging findings [1,4]. The former requires (semi) urgent surgical correction mainly to prevent infection, the latter allows calm clinical evaluation and surgical correction to release tethering $[1,4]$ or whenever in doubt about its true nature. Martinez-Lage et al. have made an excellent contribution to the literature, giving a detailed description

it penetrates the dura. d When the stalk is reflected cranially, the dural sleeve surrounding the stalk where it penetrates the dura is seen. e A thick, fibrous stalk, surrounded by some extradural fat, is attached to the conus medullaris (in this particular case close to the junction with the filum terminale). f The stalk has been cut approximately $1 \mathrm{~cm}$ below the lowest nerve roots branching off the spinal cord. The filum terminale has been cut as well. Histological examination revealed a solid stalk composed of connective tissue with some intermingled nervous and muscle tissue

of 8 children with a DS and 12 children with as DSS. However, as long as neuroradiologists, clinicians, and pathologists use different eponyms to describe both entities [1], the confusion will continue, and affected patients may be given inappropriate care. Therefore, we strongly advocate to use a uniform nomenclature, and suggest the term spinal dermal sinus (a true sinus), and spinal dermal-sinus-like stalk (a stalk rather than a sinus) [4]. 
Open Access This article is distributed under the terms of the Creative Commons Attribution Noncommercial License which permits any noncommercial use, distribution, and reproduction in any medium, provided the original author(s) and source are credited.

\section{References}

1. Martinez-Lage JF, Almagro MJ, Ferri-Niguez B, Izura Azanza V, Serrano C, Domenech E (2011) Spinal dermal sinus and pseudo- dermal sinus tracts: two different entities. Child's Nerv Syst 27:609-616

2. French BN (1983) The embryology of spinal dysraphism. Clin Neurosurg 30:295-340

3. van Aalst J, Beuls EAM, Cornips EMJ, Vanormelingen L, Vandersteen M, Weber JW, Vles JSH (2006) Anatomy and surgery of the infected dermal sinus of the lower spine. Child's Nerv Syst 22:1307-1315

4. van Aalst J, Beuls EAM, Cornips EMJ, van Straaten HWM, Boselie AFM, Rijkers K, Weber JW, Vles JSH (2009) The spinal dermal-sinus-like stalk. Child's Nerv Syst 25:191-197 